Research Article

\title{
Feasibility Study of Interlayer Slide Monitoring Using Postembedded Piezoceramic Smart Aggregates
}

\author{
Jianchao Wu $\mathbb{D}^{1,2}$ Qingzhao Kong $\mathbb{D}^{2},^{2}$ Ing Lim, ${ }^{3}$ and Gangbing Song $\mathbb{D}^{2,3}$ \\ ${ }^{1}$ Key Laboratory of Earthquake Geodesy, Institute of Seismology, China Earthquake Administration, Wuhan 430071, China \\ ${ }^{2}$ Department of Mechanical Engineering, University of Houston, Houston, TX 77204, USA \\ ${ }^{3}$ Department of Civil and Environmental Engineering, University of Houston, Houston, TX 77204, USA
}

Correspondence should be addressed to Gangbing Song; gsong@uh.edu

Received 10 August 2017; Accepted 3 January 2018; Published 25 March 2018

Academic Editor: Yinan Zhang

Copyright (c) 2018 Jianchao Wu et al. This is an open access article distributed under the Creative Commons Attribution License, which permits unrestricted use, distribution, and reproduction in any medium, provided the original work is properly cited.

Utilizing embedded transducers is an effective approach to monitor a landslide. However, for existing structures, sensors can only be postembedded, which involves drilling and grouting, and may change the original state of the structure, which calls for the need to study the effectiveness of postembedded transducers. The main focus of this paper is the feasibility study of the interlayer slide detection using postembedded piezoceramic smart aggregates (SAs). In this study, a small landslide structure that involves a weak layer is studied and two pairs of SAs were embedded in predetermined positions inside the structure. To study the difference, one pair of transducer was preembedded and the other pair was postembedded. Within each pair, one SA was employed as an actuator to generate stress waves, and another SA used as a sensor to detect wave responses. Active-sensing approach was developed to perform continuous monitoring during structural loading that was used to induce an interlayer slide. The occurrence of interlayer slide attenuates wave energy and decreases signal intensity. A wavelet-packed index was proposed to detect the occurrence and development of interlayer slide. Experimental results demonstrated that SA installation through postembedding process is an innovative yet effective approach to monitor interlayer slide.

\section{Introduction}

Landslides may cause immense property damage and even loss of life. Landslides are usually triggered by environmental changes, such as earthquake, heavy rainfall, or rise of ground water levels. These factors can amplify the inherent weakness inside a landslide body, which is defined as a weak layer [1]. Landslides occur due to interlayer slide along the weak layer. The interface between the weak layer and the surrounding medium transfers internal stress when the slope mass is subjected to vertical loading compression. Before a slide, the friction between these two regions accumulates until the accumulated stress is suddenly released and the mass slips along the weak sliding surface. It is essential to develop an effective detection technique to monitor and study interlayer slide damage to provide early warning.
Continuous and in situ monitoring of landslide is essential for the identification of potential landslide and predicting the behavior of active landslide, and its importance has been recognized by the scientific community since adequate monitoring is an effective measure for understanding kinematic aspects of landslide movement. In the past decades, different types of instruments and techniques have been utilized for landslide monitoring. These techniques can be classified as remote sensing or satellite technique [2-4], photogrammetric technique $[5,6]$, ground-based geodetic or observational technique [7-9], satellite-based geodetic technique [10-13], and geotechnical technique $[14,15]$. The selection of techniques for natural landslide monitoring depends on the different types of deformations that will affect the stability analysis. Each monitoring technique has its advantages but retains limitations. For example, the ground-based geodetic technique requires an unobstructed line of sight between 
the instrument and the targeting prism; the detection range of the remote sensing or satellite technique is costly limited to a small scale of satellite imagery; as for the photogrammetric technique, the temporal coverage is relatively poor; the geotechnical technique may lose the data if transfer media is not operating and internal storage is not activated [1].

Piezoceramic materials, with the unique advantages of wide bandwidth, low cost, small size, embeddability, and dual actuation/sensing function, have been actively researched in structural health monitoring (SHM) for many years [16-28]. The unique properties of piezoceramic materials have led to a wide variety of research, including material modeling [29-31], sensor development [32-35], energy harvesting [36-41], actuation, and sensing mechanisms [42, 43], among others. One particular piezoceramic material, lead zirconate titanate (PZT), has a strong piezoelectric effect and is commonly used. For example, a pair of PZT patches was used in smart aggregate (SA), which offers protection to the fragile PZT patches. Smart aggregates have found a wide range of active sensing enabled research, where at least two SAs are used with one as an actuator to generate stress waves and the other one as a sensor to detect the propagated waves. By interrogating the signals detected by the sensor, the changes along the wave propagating path between the actuator and the sensor can be monitored. Examples of active sensing with embedded SAs include crack and damage detection of concrete structures [44-48], water seepage monitoring of cement [49-51], soil freeze-thaw status monitoring [52], and cure-state monitoring [53]. In addition, the concept of active sensing using PZT transducers is extended to bolt looseness monitoring $[54,55]$. In a preliminary study, we demonstrated that an interlayer slide can be effectively monitored using preembedded smart aggregates with the active sensing approach [56]. In addition, this technology is a low-cost method and can determine the interface slide in real time. However, for an existing structure or landslide body, transducers, such as the smart aggregates, have to be installed through the postembedding procedure. In addition, to enable the active sensing approach, at least two SAs have to be installed with one on each side of the interlayer. The installation of postembedded smart aggregates involves drilling, embedding the smart aggregates, and grouting, and obviously the postembedding procedure changes the original conditions of the landslide body, which calls for experimental study to verify the effectiveness of the active sensing approach using embedded SAs through the postembedding procedure.

Currently, there is no study on interlayer slide monitoring with postembedded piezoceramic transducers, to the author's best knowledge. In this study, the active sensing approach using postembedded SAs was adopted as transducers for interlayer slide monitoring of a specimen with a weak layer under compressive loading. Two pairs of SAs were utilized for the excitation and detection of the sine sweep signals. For comparison, one pair of SAs was preembedded and the other pair was postembedded. The wavelet packetbased energy index of the continuous signals was employed

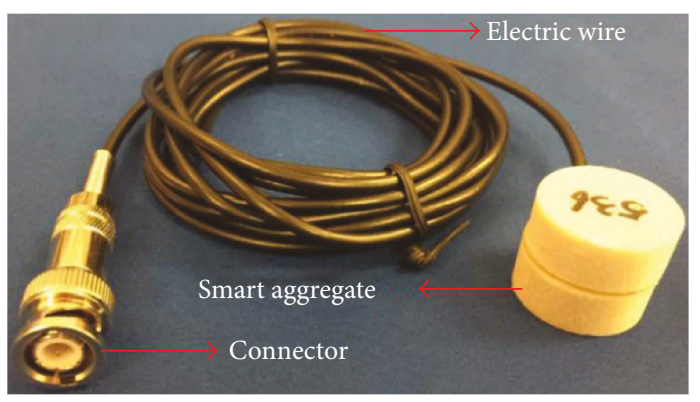

FIGURE 1: A fabricated smart aggregate.

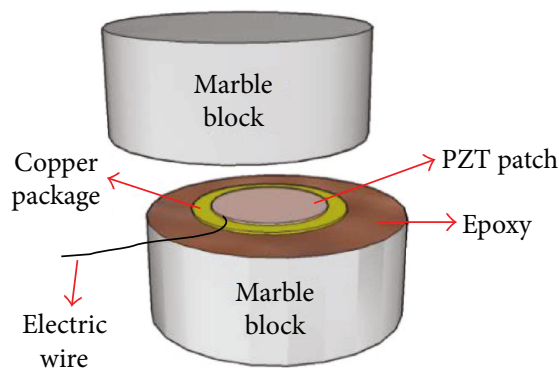

Figure 2: Components of a smart aggregate.

to quantitatively indicate the occurrence and development of the interlayer slide. Experimental results verified the feasibility and effectiveness of postembedded SAs for in situ interlayer slide detection for landslide monitoring through a comparative study.

\section{Detection Principle}

2.1. Smart Aggregate. Smart aggregates (SAs) are piezoceramicbased multifunctional transducers (Figure 1) and have been researched in structural health monitoring. SA was first proposed by Song et al. $[57,58]$ and can be embedded and fixed at a designated position within a concrete structure before casting. Due to the direct and converse piezoelectric effect of the PZT patch, SAs can satisfy the dual functions of actuation and sensing. In this paper, an SA was fabricated by sandwiching two PZT patches between a pair of cylindrical marble blocks with epoxy and proper electric shielding, as shown in Figure 2.

2.2. Principle of the Active Sensing Method. In this study, a smart aggregate-based active sensing approach is used to detect the interlayer slide damage. Active sensing method takes advantage of the dual actuation/sensing capability of piezoelectric transducers. Figure 3 demonstrates the SAbased active sensing approach for interlayer slide detection. Two pairs of SAs are used, and each pair is installed in opposite sides of a weak interlayer. Between them, one pair of SAs was preembedded before the casting, and the other pair was postembedded after curing of the concrete. For each pair, one SA functions as an actuator to generate stress waves which propagate along the structure and across the 


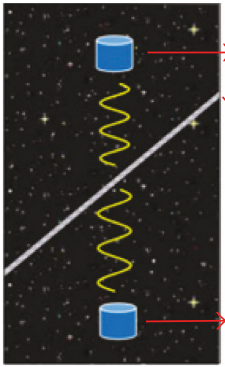

(a)
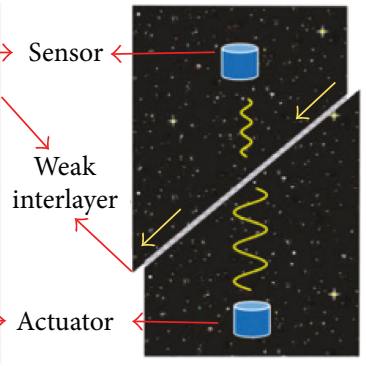

(b)
FIGURE 3: Schematic diagram of smart aggregate-based active sensing approach for interlayer slide detection. (a) Before slide. (b) After slide.

interlayer, and the other one acts as a sensor to detect the wave response. The energy attenuation of the propagating wave crossing the interlayer is heavily influenced by the interlayer condition. If an interlayer slide occurs, which weakens the interlayer, the energy attenuation of the stress wave will increase. Analysis of the sensor signal can reveal the occurrence of the interlayer slide when compared with a baseline or initial condition.

2.3. Interlayer Slide Index. Wavelet packet analysis was used as a signal-processing tool to analyze the detected signal. A wavelet is a waveform of effective limited duration with an average value of zero. Wavelet packet analyses enable the inspection of narrow frequency bands over a relatively short time window [59]. Based on wavelet packet analysis, an interlayer slide index, which represents the transmission energy loss caused by the development of slide failure, can be established as follows:

The sensor signal $X$, which represents the detected wave response, is first filtered by a Butterworth filter and then decomposed by an $n$-level wavelet packet decomposition into $2^{n}$ signal sets $\left\{X_{1}, X_{2}, \ldots, X_{j}, \ldots, X_{2}^{n}\right\} . X_{j}$ is the decomposed sensor signal and can be represented as

$$
X_{j}=\left[X_{j, 1}, X_{j, 2}, \ldots, X_{j, m}\right] \text {, }
$$

where $m$ is the number of the sampling data and $j$ is the frequency band number $\left(j=1,2, \ldots, 2^{n}\right)$.

The energy of each decomposed signal $E_{j}$ is defined as

$$
E_{j}=X_{j, 1^{2}}+X_{j, 2^{2}}+\cdots+X_{j, m^{2}} .
$$

The energy vector of the signal $X_{i}$ at the $i$ th measurement is given by

$$
\mathbf{E}_{i, i}=\left[E_{i, 1}, E_{i, 2}, \ldots, E_{i, 2^{n}}\right] .
$$

The interlayer slide index, using root-mean-square deviation (RMSD) [60-62], indicates the severity of damage between the chosen actuator-sensor systems. Specifically, the index can be developed by calculating the RMSD between the energy vectors of the initial state and subsequent states

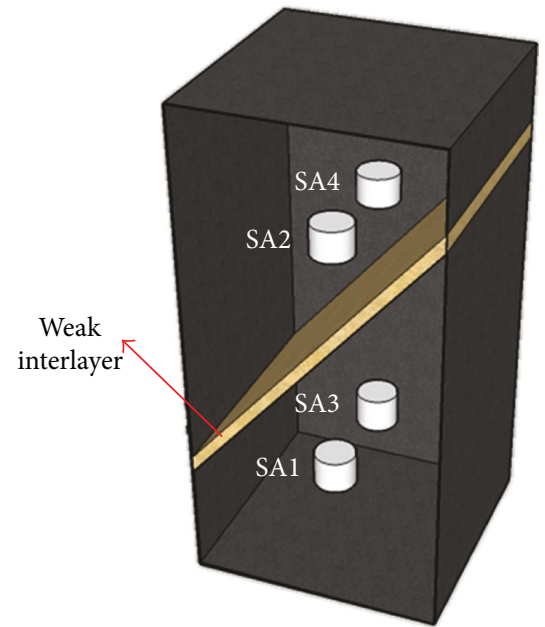

FIgURE 4: Schematics of the specimen. SA1 and SA2 are preembedded. SA3 and SA4 are postembedded.

during the test. The proposed wavelet-based interlayer slide index at the $i$ th time index is defined as

$$
I=\sqrt{\frac{\sum_{j=1}^{2^{n}}\left(E_{i, j}-E_{h, j}\right)^{2}}{\sum_{j=1}^{2^{n}}\left(E_{h, j}\right)^{2}}},
$$

where $E_{h, j}$ is the energy at the $j$ th frequency band $(j=1,2, \ldots$, $2^{n}$ ) in the initial or the healthy state and $E_{i, j}$ is the energy in the $i$ th time index. As an example, for a laboratory test of the structure with an interlayer, the structure is considered in a healthy state without the slide before the compressive loading, and the interlayer slide index value is zero at this situation. As the loading progresses, a slide happens and the index value will eventually approach one. Therefore, the values of the interlayer slide index can be used to monitor the interlayer slide, since the interlayer slide index was computed based on the baseline data which was recorded when the structure is in health status. The method has the capability to tolerate small degrees of misalignment when the smart aggregate pairs were postembedded in the structure.

\section{Experimental Setup}

The specimen and SAs were fabricated in the Smart Material and Structure Laboratory, University of Houston. To verify the feasibility and reliability of our proposed approach, we chose mortar to simulate the environmental medium due to its uniform and relative isotropic properties so that the experimental results will not be strongly affected by the nonlinear and nonisotropic material properties of the medium. In addition, to implement the active sensing approach, two pairs of smart aggregates are embedded into the specimen at prearranged locations on each side of the interlayer. The size of the specimen was $150 \times 150 \times 300 \mathrm{~mm}^{3}$, and the location of SAs is shown in Figure 4. The size was selected to avoid mechanical damage to the surrounding medium. Considering that the natural landslide usually occurs in high inclination, the angle between the weak interlayer and 
TABLE 1: Composition of the surrounding specimen and weak interlayer under study.

\begin{tabular}{lcc}
\hline Material & Constituent & Content $(\mathrm{kg})$ \\
\hline \multirow{3}{*}{ Weak interlayer } & Mortar & 0.25 \\
& Clay & 0.075 \\
& Sand & 0.075 \\
& Water & 0.1 \\
\hline \multirow{2}{*}{ Specimen } & Mortar & 3.85 \\
& Water & 0.65 \\
\hline
\end{tabular}

horizontal surface was set to $40^{\circ}$. In this research, a compression test was designed to simulate the interfacial slide of the specimen. It should be noted that the thickness of the weak interlayer was $15 \mathrm{~mm}$. The weak interlayer was simulated by a mixture of mortar, clay, sand, and water, and the mixing ratio was mortar : clay: sand $:$ water $=5: 1.5: 1.5: 2$. Its quantitative composition is listed in Table 1 . The dimensional details are shown in Figure 5.

The preembedded SAs were labeled SA1 and SA2, while the postembedded SAs were labeled SA3 and SA4. Postembedded SAs were installed by drilling a $30 \mathrm{~mm}$ diameter and $35 \mathrm{~mm}$ deep hole in the side face of the specimen, as shown in Figure 6. The SAs were embedded into the hole and refilled by nonshrinkage mortar. Both preembedded and postembedded SAs were located symmetrically at the same level with a horizontal distance of about $75 \mathrm{~mm}$. To ensure the position and orientation of the smart aggregates, the first smart aggregate was installed after the mortar was poured at the designed height where the first smart aggregate should be located. With a 5-minute hold for the mortar drying and a careful check of the position and orientation of the smart aggregate, more mortar was filled until the second smart aggregate was placed. Finally, the mortar was poured to completely fill the drilled hole. After the curing of the mortar, the two SAs became an integrated part of the landslide body. The SA1 and SA3 were used as the actuators to generate repeated swept sine wave. SA2 and SA4 were adopted as the sensors to detect the wave response.

Instrumentation includes two power amplifiers (Trek 2100HF), data acquisition system (NI USB6353), and a Shore Western universal hydraulic testing machine, as shown in Figure 7. The loading test was conducted by employing the hydraulic testing machine with a separate acquisition system. During the test, a swept sine wave was generated by the NI USB6353 system and then amplified by the power amplifier with a gain of 50 . The frequency range of the swept sine wave is from $100 \mathrm{~Hz}$ to $150 \mathrm{kHz}$. The amplitude and period of the signal are $150 \mathrm{~V}$ and 1 second, respectively. The sampling rate of the data acquisition system is $1.25 \mathrm{MS} / \mathrm{s}$.

Loading on the specimen was achieved with an $1112 \mathrm{KN}$ capacity Shore Western universal hydraulic testing machine. The specimen was subjected to an axial pressure loading, with a loading rate of $0.033 \mathrm{~mm} / \mathrm{min}$. Every 10 minutes, SA1 and SA3 generated repeated swept sine waves, and the corresponding sensors (SA2 and SA4) detected the wave signal that propagated through the weak interlayer.

\section{Experimental Results and Discussions}

4.1. Pressure Loading History. The loading test lasted 310 minutes, and the loading history curve is shown in Figure 8 . The entire loading history can be divided into three stages. During the first stage (0-60 minutes), the loading history curve displayed a linear increase which indicated that the structure experienced elastic deformation and becomes denser. During the second stage (61-290 minutes), the loading history curve increased linearly. With an increasing load, the friction between the weak interlayer and the surrounding medium accumulated and increased. During the last stage (291-310 minutes), the loading stress suddenly decreased, which indicated the occurrence of the interlayer slide. In this stage, a relative displacement was observed between the upper specimen and the lower specimen.

4.2. Time Domain Analysis. The detected signals before and after the interlayer slide in time domain by SA2 and SA4 are shown in Figure 9. In each figure, the red and blue curves represented the signal received at 290 and 300 minutes, respectively. The entire signals corresponded to one period of the excitation signal. Due to the differences of the location, interface, wave propagation medium, and orientation between the preembedded SA pair and the postembedded SA pair, the received signals of these two pairs present similar amplitude levels but still retain unique details. Both of the received signal amplitudes experienced obvious decreases after slide damage occurs due to the attenuation of the stress wave that propagated through the sliding interlayer. From the experimental results of the time domain signal response, the postembedded SAs show potentials to be used for the detection of the interface slide.

4.3. Wavelet Packet-Based Analysis. According to the wavelet decomposition, a signal can be decomposed into several frequency bands. As shown in Figures 10 and 11, energy vectors (level 5 decomposition) of the time domain signal given in Figure 9 are formed by calculating the energy of each subset (frequency band) to present the energy distribution along the frequency bands. When the interlayer slide occurred, the propagating stress wave energy attenuates at the sliding interface so that to reduce the energy of the signal received by SA sensors. Compared with the energy vector before and after the slide as shown in Figures 10 and 11, obvious energies' drop can be found at different frequency bands. It can be seen that the wavelet-based analysis helps to provide quantitative attenuation values of the signal corresponding to each frequency band before and after the interlayer slide. To further determine the occurrence of the interface slide, the wavelet packet-based interlayer slide damage index is computed, as shown in the next section.

4.4. Wavelet Packet-Based Interlayer Slide Damage Indices. To provide quantitative analysis of the entire loading process, the energy of the detected signal was computed by employing the wavelet packet-based energy analysis. The computed interlayer slide damage indices are shown in Figures 12 and 13. Both figures depict the energy levels throughout the entire loading process. Both indices show a similar overall 


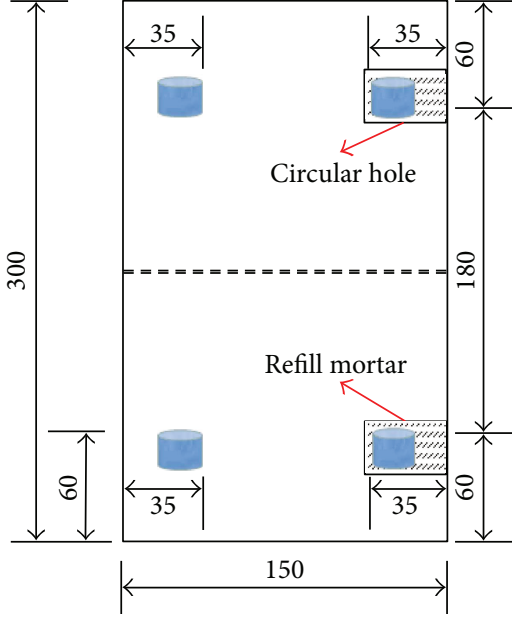

(a)

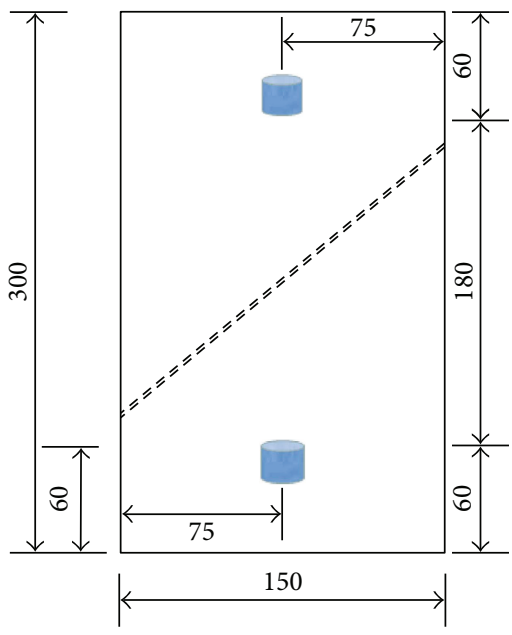

(b)

FiguRE 5: Locations of smart aggregates in the specimen (unit: mm). (a) Lateral view. (b) Front view.

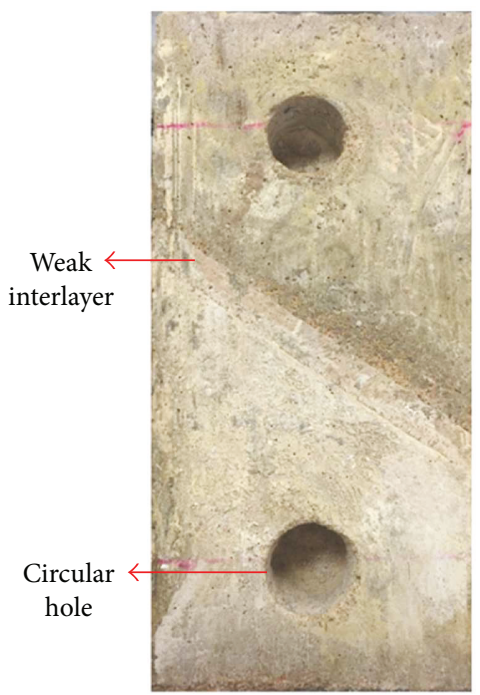

(a)

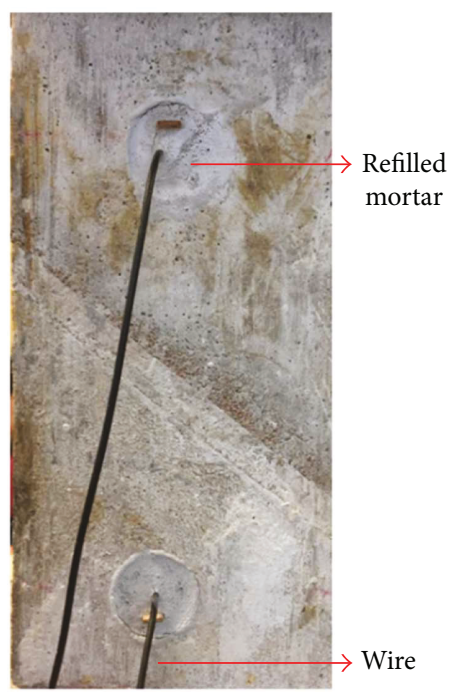

(b)

FIGURE 6: Installation of postembedded smart aggregates in the specimen. (a) Circular hole before installation of SA. (b) Refilled mortar after the installation of SAs.

increasing trend, and a sharp increase was observed near the 300th minute, due to the occurrence of interlayer slide damage that resulted in a sudden release of stress wave energy. Correspondingly, the damage indices increased suddenly when the slide occurred near the 300th minute. Both pre- and postembedded SAs effectively detected the interlayer slide.

We monitored the occurrence of the interlayer slide in real time by detecting the sensors' responses and the associated energy levels. Compared to the time domain analysis, wavelet packet-based energy analysis shows an abrupt energy change that corresponded to the interlayer slide. Therefore, wavelet packet-based energy analysis using the data from the postembedded transducers has the capacity to determine the occurrence of an interlayer slide.
4.5. Discussions. Through the experiment, the occurrence of the interlayer slide was successfully detected in real time by employing the active sensing-based approach with postembedded smart aggregates. It should be noted that the deformation of the specimen with a weak interlayer under loading compression is complex. The structure is a heterogeneous material and exhibits a complex elastic behavior related to the presence of microcracks. Due to the complexity of the interlayer slide, there is lack of real-time-based technologies to monitor the interlayer slide, especially at its early age. This research proposes a piezoceramic-based active sensing approach which could have a potential to provide an early warning of the interlayer slide in real time. Due to the principle of the proposed method, the energy of the propagating stress wave between SAs attenuates (a sudden drop of 


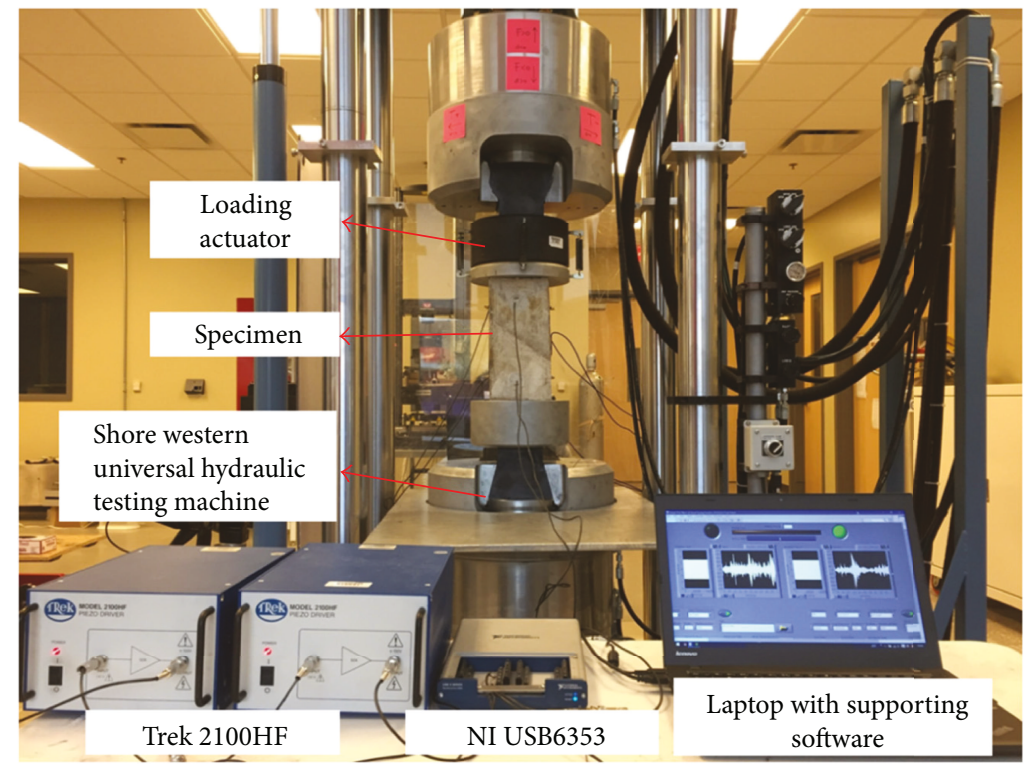

Figure 7: Experimental setup.

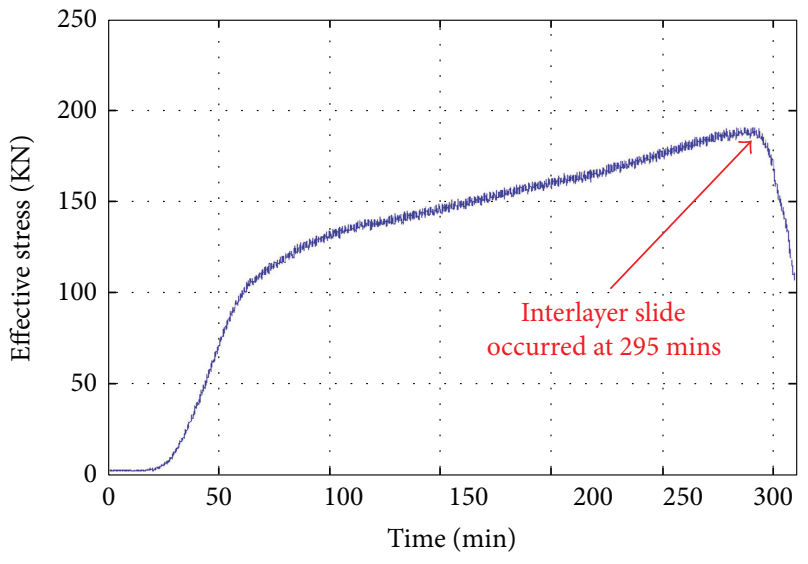

Figure 8: Loading history.

the received energy) when the slide occurs. By using the developed sliding interlayer index, the slide event can be immediately determined when a "sharp" increase value is presented in the index. However, many factors including the distance of SAs, wave propagation medium property, and excitation signal parameters, may influence the threshold values. A comparative study of the results of preembedded smart aggregates and postembedded smart aggregates reveals that the differences between the two are very limited, demonstrating the effectiveness of interlayer slide detection by using the postembedded smart aggregates. Since the proposed method is limited to detecting a local slide near the sliding surface, a number of actuator-and-sensor pairs should be deployed to monitor the slide in an actual landslide body. Compared with other landslide monitoring techniques, the proposed active sensing approach based on SAs is a low-cost, robust, and real-time approach and can provide a localized damage information. Due to these advantages, the smart aggregates can be easily postembedded into the existing landslide body. Currently, the proposed technique is still a qualitative method, which can hardly provide accurate parameters of the landslide.

According to the acoustoelastic theory, the velocity of the propagating ultrasonic waves varies in the concrete body subjected to a high level of stress [63]. The differences of the wave velocities will affect the wave propagation in concrete. In this research, the acoustoelastic effect during the loading test is not considered. Further analysis of the results considering the acoustoelastic effect will be investigated. In addition, more tests will be conducted to further verify the validity, reliability, and accuracy of the proposed approach. The accuracy of the proposed approach on the detection of the interlayer slide damage of a structure due to bending force will also be considered.

\section{Conclusions}

This research, through experimental means, demonstrated that postembedded smart aggregate-based technique for interlayer slide monitoring was feasible and effective in detecting a slide damage. This technique adopts a stress wave-based active sensing approach with piezoceramic transducers. Since a slide event weakened the interface between the two layers and reduced the energy carried by the stress wave crossing the interlayer, the occurrence of the interlayer slide was detected when a significant drop in the detected signal was observed. It was found that both pre- and postembedded SAs effectively detected interlayer slide damage with minimum differences. In addition, the proposed wavelet packet-based interlayer slide indices can qualitatively determine the initiation and development of a slide damage. The index values provided by postembedded SAs reflect the occurrence of the interlayer slide and are consistent with time domain results. In conclusion, the postembedded smart aggregate-based method has the potential for implementation to monitor and detect landslide; however, the determination of the threshold value of the developed sliding 


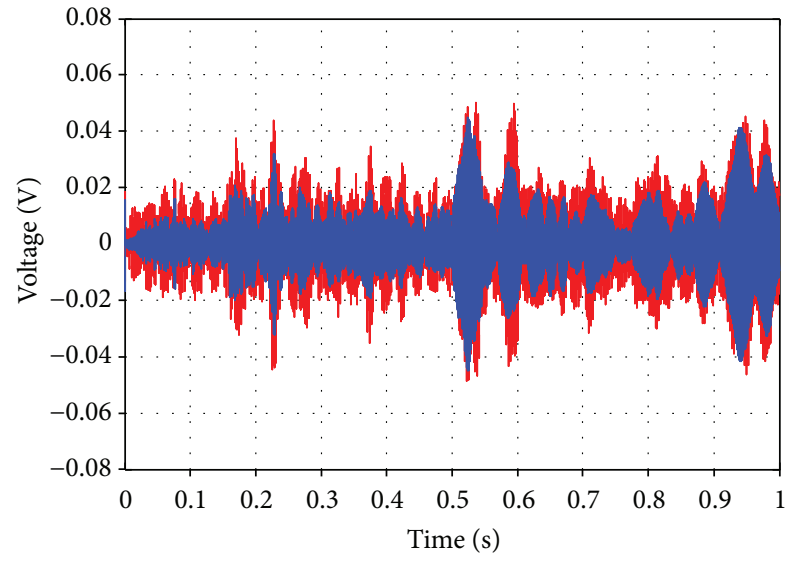

- Signal received at 290th minute

— Signal received at 300th minute

(a) SA2

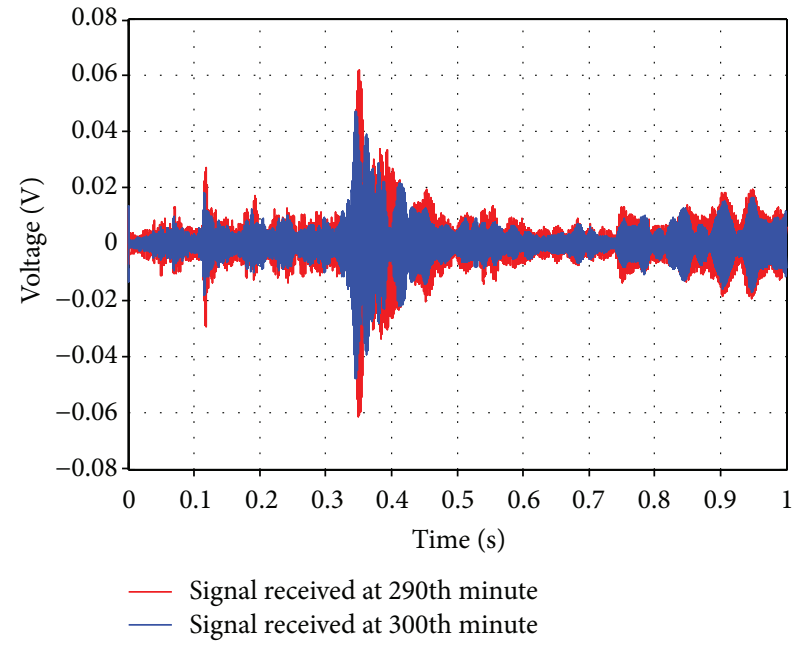

(b) SA4

FIgURE 9: Signal received by preembedded SAs before and after slide.

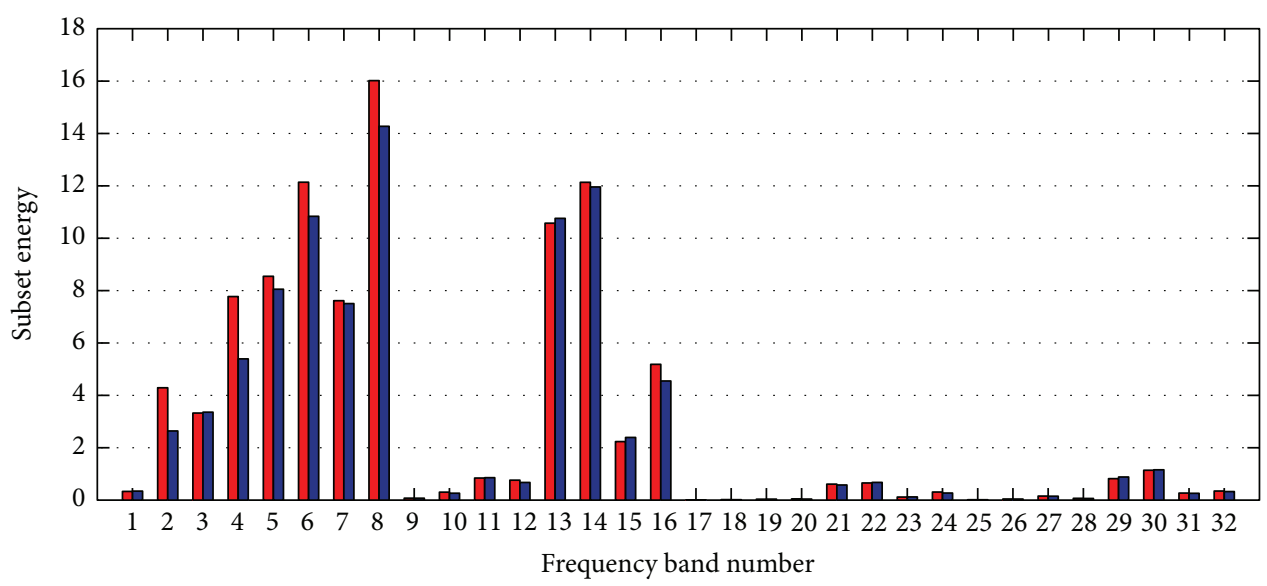

Signal received at 290th minute

Signal received at 300th minute

FIGURE 10: Energy vector of the signals received by postembedded SA2 before and after slide.

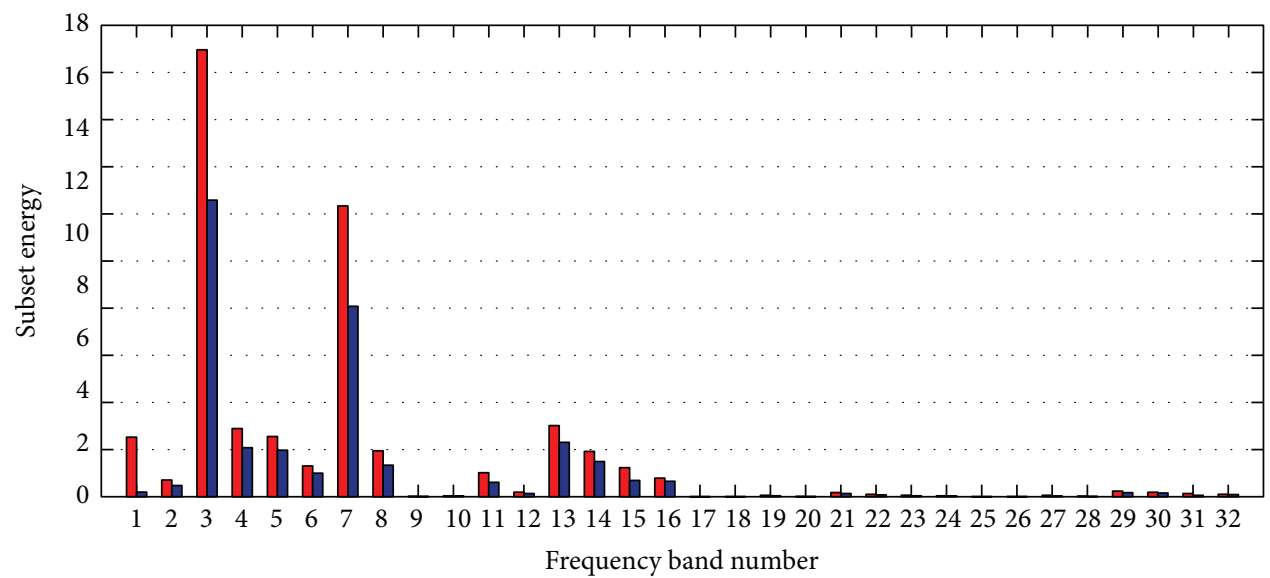

Signal received at 290th minute

Signal received at 300th minute

FIGURE 11: Energy vector of the signals received by postembedded SA2 before and after slide. 


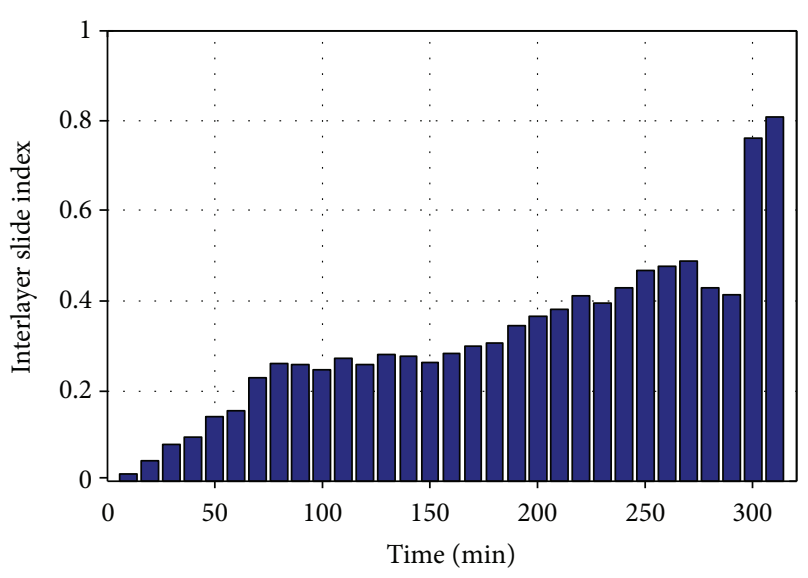

FIGURE 12: Interlayer slide indices of preembedded SA2 throughout the test.

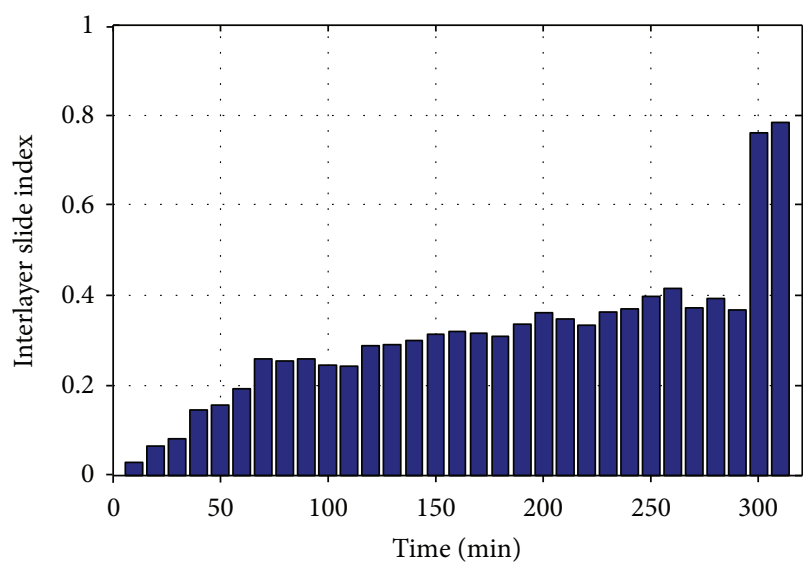

FIGURE 13: Interlayer slide index of postembedded SA4 throughout the test.

interlayer index requires further theoretical and experimental investigation, which will be considered in the authors' future work.

\section{Conflicts of Interest}

The authors declare no conflict of interest.

\section{Authors' Contributions}

Jianchao Wu and Gangbing Song developed the idea, designed the experiments, and wrote the paper. Qingzhao Kong helped design the experiment and write the paper. Ing Lim helped perform the experiments. Gangbing Song made critical comments to the paper. Jianchao $\mathrm{Wu}$ and Qingzhao Kong contributed equally to this work.

\section{Acknowledgments}

This work is supported by the Director Foundation of the Institute of Seismology, China Earthquake Administration (IS201726163).

\section{References}

[1] P. Savvaidis, "Existing landslide monitoring systems and techniques," in From Stars to Earth and Culture. In Honor of the Memory of Professor Alexandros Tsioumis, pp. 242-258, The Aristotle University of Thessaloniki, Greece, 2003.

[2] D. Tarchi, N. Casagli, R. Fanti et al., "Landslide monitoring by using ground-based SAR interferometry: an example of application to the Tessina landslide in Italy," Engineering Geology, vol. 68, no. 1-2, pp. 15-30, 2003.

[3] X. Ye, H. Kaufmann, and X. F. Guo, "Landslide monitoring in the Three Gorges area using D-InSAR and corner reflectors," Photogrammetric Engineering \& Remote Sensing, vol. 70, no. 10, pp. 1167-1172, 2004.

[4] C. Colesanti and J. Wasowski, "Investigating landslides with space-borne synthetic aperture radar (SAR) interferometry," Engineering Geology, vol. 88, no. 3-4, pp. 173-199, 2006.

[5] G. Bitelli, M. Dubbini, and A. Zanutta, "Terrestrial laser scanning and digital photogrammetry techniques to monitor landslide bodies," International Archives of Photogrammetry, Remote Sensing and Spatial Information Sciences, vol. 35, no. B5, pp. 246-251, 2004.

[6] E. Brückl, F. K. Brunner, and K. Kraus, "Kinematics of a deep-seated landslide derived from photogrammetric, GPS and geophysical data," Engineering Geology, vol. 88, no. 3-4, pp. 149-159, 2006.

[7] S. C. Stiros, C. Vichas, and C. Skourtis, "Landslide monitoring based on geodetically derived distance changes," Journal of Surveying Engineering, vol. 130, no. 4, pp. 156162, 2004.

[8] M. Haberler-Weber, "Analysis and interpretation of geodetic landslide monitoring data based on fuzzy systems," Natural Hazards and Earth System Science, vol. 5, no. 5, pp. 755-760, 2005.

[9] G. Barla, F. Antolini, M. Barla, E. Mensi, and G. Piovano, "Monitoring of the Beauregard landslide (Aosta Valley, Italy) using advanced and conventional techniques," Engineering Geology, vol. 116, no. 3-4, pp. 218-235, 2010.

[10] J. A. Gili, J. Corominas, and J. Rius, "Using Global Positioning System techniques in landslide monitoring," Engineering Geology, vol. 55, no. 3, pp. 167-192, 2000.

[11] C. Squarzoni, C. Delacourt, and P. Allemand, "Differential single-frequency GPS monitoring of the La Valette landslide (French Alps)," Engineering Geology, vol. 79, no. 3-4, pp. 215-229, 2005.

[12] G. Wang, "Millimeter-accuracy GPS landslide monitoring using precise point positioning with single receiver phase ambiguity (PPP-SRPA) resolution: a case study in Puerto Rico," Journal of Geodetic Science, vol. 3, no. 1, pp. 22-31, 2013.

[13] L. Gui, K. Yin, and T. Glade, "Landslide displacement analysis based on fractal theory, in Wanzhou District, Three Gorges Reservoir, China," Geomatics, Natural Hazards and Risk, vol. 7, no. 5, pp. 1707-1725, 2016.

[14] J. Corominas, J. Moya, A. Lloret et al., "Measurement of landslide displacements using a wire extensometer," Engineering Geology, vol. 55, no. 3, pp. 149-166, 2000.

[15] L. Simeoni and L. Mongiovì, "Inclinometer monitoring of the Castelrotto landslide in Italy," Journal of Geotechnical and Geoenvironmental Engineering, vol. 133, no. 6, pp. 653-666, 2007. 
[16] B. Sabet Divsholi and Y. Yang, "Combined embedded and surface-bonded piezoelectric transducers for monitoring of concrete structures," NDT \& E International, vol. 65, pp. 2834,2014 .

[17] C. Dumoulin, G. Karaiskos, J. Carette, S. Staquet, and A. Deraemaeker, "Monitoring of the ultrasonic P-wave velocity in early-age concrete with embedded piezoelectric transducers," Smart Materials and Structures, vol. 21, no. 4, article 047001, 2012.

[18] Z. Shi and T. Zhang, "Bending analysis of a piezoelectric curved actuator with a generally graded property for the piezoelectric parameter," Smart Materials and Structures, vol. 17, no. 4, article $045018,2008$.

[19] V. P. Venugopal and G. Wang, "Modeling and analysis of Lamb wave propagation in a beam under lead zirconate titanate actuation and sensing," Journal of Intelligent Material Systems and Structures, vol. 26, no. 13, pp. 1679-1698, 2015.

[20] Y. Yang, V. G. M. Annamdas, C. Wang, and Y. Zhou, “Application of multiplexed FBG and PZT impedance sensors for health monitoring of rocks," Sensors, vol. 8, no. 1, pp. 271289, 2008.

[21] W. Dansheng, L. Zhi, and Z. Hongping, "A new threedimensional electromechanical impedance model for an embedded dual-PZT transducer," Smart Materials and Structures, vol. 25, no. 7, article 075002, 2016.

[22] D. Wang, Q. Wang, H. Wang, and H. Zhu, "Experimental study on damage detection in timber specimens based on an electromechanical impedance technique and RMSD-based Mahalanobis distance," Sensors, vol. 16, no. 10, p. 1765, 2016.

[23] C. Dumoulin and A. Deraemaeker, "Design optimization of embedded ultrasonic transducers for concrete structures assessment," Ultrasonics, vol. 79, pp. 18-33, 2017.

[24] Z. F. Shi, H. J. Xiang, and B. F. Spencer Jr, "Exact analysis of multi-layer piezoelectric/composite cantilevers," Smart Materials and Structures, vol. 15, no. 5, pp. 1447-1458, 2006.

[25] G. Song, H. Li, B. Gajic, W. Zhou, P. Chen, and H. Gu, "Wind turbine blade health monitoring with piezoceramic-based wireless sensor network," International Journal of Smart and Nano Materials, vol. 4, no. 3, pp. 150-166, 2013.

[26] Z. Wu and M. Abe, "Structural health monitoring and intelligent infrastructure," in Proceedings of the First International Conference on Structural Health Monitoring and Intelligent Infrastructure, 13-15 November 2003, Tokyo, Japan, Taylor \& Francis, 2003.

[27] S. V. Gopinathan, V. V. Varadan, and V. K. Varadan, "A review and critique of theories for piezoelectric laminates," Smart Materials and Structures, vol. 9, no. 1, pp. 24-48, 2000.

[28] X. Wang, L. Ye, Y.-W. Mai, and S. C. Galea, "Designing for piezoelectric ceramic wafers bonded on structures using force transfer criteria," Smart Materials and Structures, vol. 9, no. 2, pp. 157-162, 2000.

[29] M. Kamlah and Q. Jiang, "A constitutive model for ferroelectric PZT ceramics under uniaxial loading," Smart Materials and Structures, vol. 8, no. 4, pp. 441-459, 1999.

[30] S. C. Hwang, C. S. Lynch, and R. M. McMeeking, "Ferroelectric/ferroelastic interactions and a polarization switching model," Acta Metallurgica et Materialia, vol. 43, no. 5, pp. 2073-2084, 1995.

[31] M. Kamlah, "Ferroelectric and ferroelastic piezoceramics modeling of electromechanical hysteresis phenomena,"
Continuum Mechanics and Thermodynamics, vol. 13, no. 4, pp. 219-268, 2001.

[32] X.-F. Li, X.-L. Peng, and K. Y. Lee, "The static response of functionally graded radially polarized piezoelectric spherical shells as sensors and actuators," Smart Materials and Structures, vol. 19, no. 3, article 035010, 2010.

[33] H. Miao, S. Dong, and F. Li, "Excitation of fundamental shear horizontal wave by using face-shear (d36) piezoelectric ceramics," Journal of Applied Physics, vol. 119, no. 17, article 174101, 2016.

[34] J. Kim and J.-H. Lee, "Self-moving cell linear motor using piezoelectric stack actuators," Smart Materials and Structures, vol. 14, no. 5, pp. 934-940, 2005.

[35] T. T. Zhang, Z. F. Shi, and B. F. Spencer Jr, "Vibration analysis of a functionally graded piezoelectric cylindrical actuator," Smart Materials and Structures, vol. 17, no. 2, article 025018, 2008.

[36] H. S. Kim, J.-H. Kim, and J. Kim, "A review of piezoelectric energy harvesting based on vibration," International Journal of Precision Engineering and Manufacturing, vol. 12, no. 6, pp. 1129-1141, 2011.

[37] J. Liang and W.-H. Liao, "Energy flow in piezoelectric energy harvesting systems," Smart Materials and Structures, vol. 20, no. 1, article 015005, 2010.

[38] Y. C. Shu, I. C. Lien, and W. J. Wu, "An improved analysis of the SSHI interface in piezoelectric energy harvesting," Smart Materials and Structures, vol. 16, no. 6, pp. 2253-2264, 2007.

[39] H. J. Song, Y. T. Choi, G. Wang, and N. M. Wereley, "Energy harvesting utilizing single crystal PMN-PT material and application to a self-powered accelerometer," Journal of Mechanical Design, vol. 131, no. 9, article 091008, 2009.

[40] F. Ewere, G. Wang, and B. Cain, "Experimental investigation of galloping piezoelectric energy harvesters with square bluff bodies," Smart Materials and Structures, vol. 23, no. 10, article 104012, 2014.

[41] H. A. Sodano, A. Erturk, J. M. Renno, and D. J. Inman, "Modeling of piezoelectric energy harvesting from an Lshaped beam-mass structure with an application to UAVs," Journal of Intelligent Material Systems and Structures, vol. 20, no. 5, pp. 529-544, 2009.

[42] Y.-H. Hsu and C.-K. Lee, "Targeted origin placement for the autonomous gain-phase tailoring of piezoelectric sensors," Smart Materials and Structures, vol. 11, no. 3, pp. 444-458, 2002.

[43] W. J. Staszewski, "Structural health monitoring using guided ultrasonic waves," in Advances in Smart Technologies in Structural Engineering. Computational Methods in Applied Sciences, vol 1, J. Holnicki-Szulc and C. M. Soares, Eds., pp. 117-162, Springer, Berlin, Heidelberg, 2004.

[44] Q. Kong, R. Robert, P. Silva, and Y. Mo, "Cyclic crack monitoring of a reinforced concrete column under simulated pseudodynamic loading using piezoceramic-based smart aggregates," Applied Sciences, vol. 6, no. 11, p. 341, 2016.

[45] L. F. Li, B. Xu, and J. M. Chen, "Damage monitoring for a Rc shear wall under rapid cyclic loading with embedded smart aggregates," Proceedings of the Eleventh International Symposium on Structural Engineering, vol. I and Ii, pp. 1432-1437, 2010.

[46] S. Gupta, J. Gonzalez, and K. J. Loh, "Damage detection using smart concrete engineered with nanocomposite cementaggregate interfaces," Structural Health Monitoring 2015: 
System Reliability for Verification and Implementation, Vols. 1 and 2, pp. 3033-3040, 2015, http://www.dpi-proceedings.com/ index.php/SHM2015/article/view/1037.

[47] C. Zhang, X. Yu, L. Alexander, Y. Zhang, R. Rajamani, and N. Garg, "Piezoelectric active sensing system for crack detection in concrete structure," Journal of Civil Structural Health Monitoring, vol. 6, no. 1, pp. 129-139, 2016.

[48] Q. Kong, Q. Feng, and G. Song, "Water presence detection in a concrete crack using smart aggregates," International Journal of Smart and Nano Materials, vol. 6, no. 3, pp. 149$161,2015$.

[49] D. Zou, T. Liu, Y. Huang, F. Zhang, C. Du, and B. Li, "Feasibility of water seepage monitoring in concrete with embedded smart aggregates by $\mathrm{P}$-wave travel time measurement," Smart Materials and Structures, vol. 23, no. 6, article 067003, 2014.

[50] Q. Feng, Q. Kong, L. Huo, and G. Song, "Crack detection and leakage monitoring on reinforced concrete pipe," Smart Materials and Structures, vol. 24, no. 11, article 115020, 2015.

[51] T. Liu, Y. Huang, D. Zou, J. Teng, and B. Li, "Exploratory study on water seepage monitoring of concrete structures using piezoceramic based smart aggregates," Smart Materials and Structures, vol. 22, no. 6, article 065002, 2013.

[52] Q. Kong, R. Wang, G. Song, Z. J. Yang, and B. Still, “Monitoring the soil freeze-thaw process using piezoceramic-based smart aggregate," Journal of Cold Regions Engineering, vol. 28, no. 2, article 06014001, 2014.

[53] K. Chung, S. Kharkovsky, Q. Kong, and G. Song, "Cure-state monitoring of concrete and mortar specimens using smart aggregates," in 2014 IEEE International Instrumentation and Measurement Technology Conference (I2MTC) Proceedings, pp. 304-308, Montevideo, Uruguay, May 2014.

[54] H. Yin, T. Wang, D. Yang, S. Liu, J. Shao, and Y. Li, “A smart washer for bolt looseness monitoring based on piezoelectric active sensing method," Applied Sciences, vol. 6, no. 11, p. 320, 2016.

[55] J. Shao, T. Wang, H. Yin, D. Yang, and Y. Li, "Bolt looseness detection based on piezoelectric impedance frequency shift," Applied Sciences, vol. 6, no. 10, p. 298, 2016.

[56] J. Wu, Q. Kong, W. Li, I. Lim, and G. Song, "Interlayer slide detection using piezoceramic smart aggregates based on active sensing approach," IEEE Sensors Journal, vol. 17, no. 19, pp. 6160-6166, 2017.

[57] G. Song, H. Gu, and Y.-L. Mo, "Smart aggregates: multifunctional sensors for concrete structures-a tutorial and a review," Smart Materials and Structures, vol. 17, no. 3, article 033001, 2008.

[58] G. Song, H. Gu, and Y. L. Mo, "Smart aggregates: a distributed intelligent multi-purpose sensor network (DIMSN) for civil structures," in 2007 IEEE International Conference on Networking, Sensing and Control, pp. 775-780, London, UK, April 2007.

[59] B. Xu, T. Zhang, G. Song, and H. Gu, "Active interface debonding detection of a concrete-filled steel tube with piezoelectric technologies using wavelet packet analysis," Mechanical Systems and Signal Processing, vol. 36, no. 1, pp. 7-17, 2013.

[60] G. Song, H. Gu, Y. L. Mo, T. T. C. Hsu, and H. Dhonde, "Concrete structural health monitoring using embedded piezoceramic transducers," Smart Materials and Structures, vol. 16, no. 4, pp. 959-968, 2007.

[61] L. Yabin, L. Dongsheng, P. Seyed Mohammad, K. Qingzhao, L. Ing, and S. Gangbing, "Bond-slip detection of concreteencased composite structure using electro-mechanical impedance technique," Smart Materials and Structures, vol. 25, no. 9, article 095003, 2016.

[62] T. Jiang, Q. Kong, W. Wang, L. Huo, and G. Song, "Monitoring of grouting compactness in a post-tensioning tendon duct using piezoceramic transducers," Sensors, vol. 16, no. 8, p. 1343, 2016.

[63] I. Lillamand, J.-F. Chaix, M.-A. Ploix, and V. Garnier, "Acoustoelastic effect in concrete material under uni-axial compressive loading,” NDT \& E International, vol. 43, no. 8, pp. 655-660, 2010. 


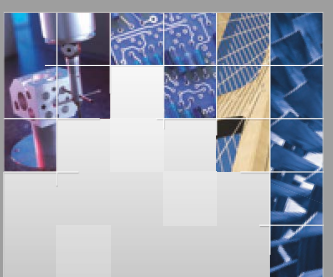

\section{Enfincering}
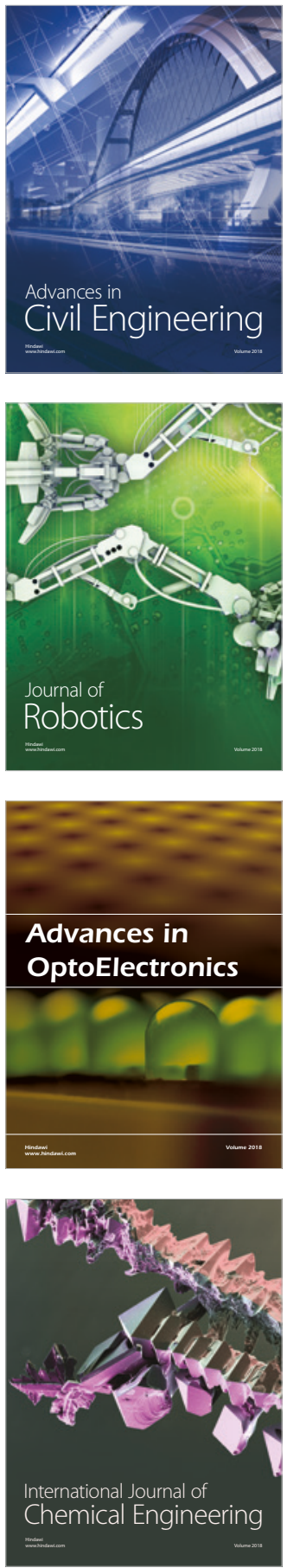

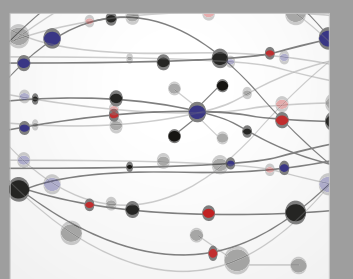

\section{Rotating \\ Machinery}

The Scientific World Journal

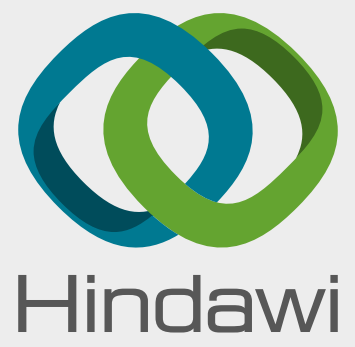

Submit your manuscripts at

www.hindawi.com
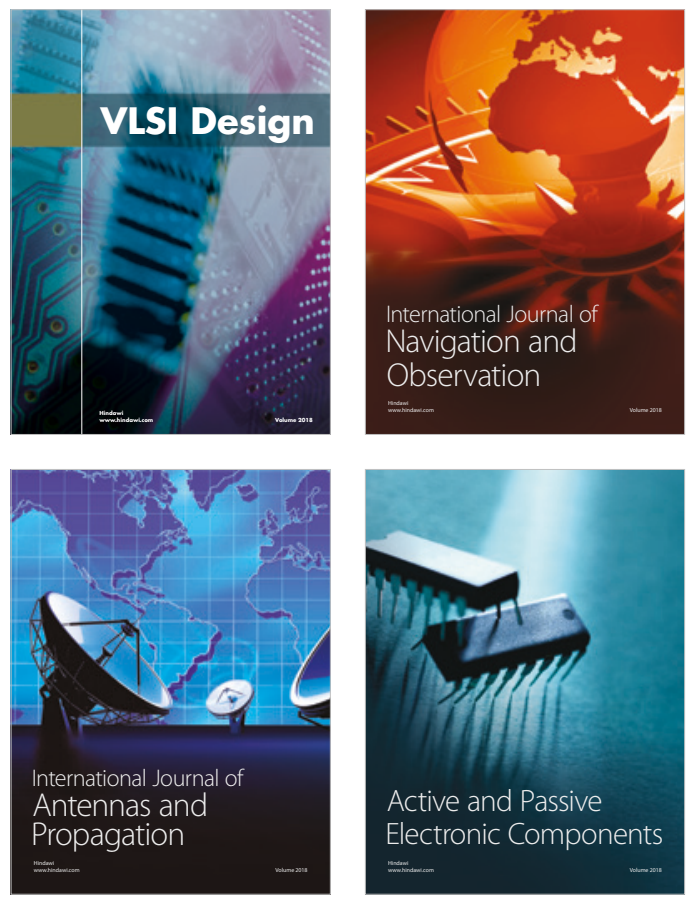
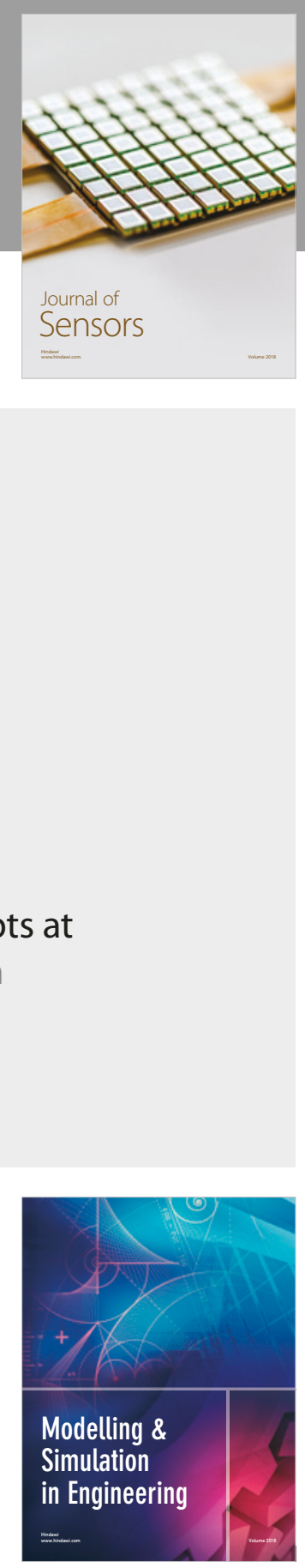

\section{Advances \\ Multimedia}
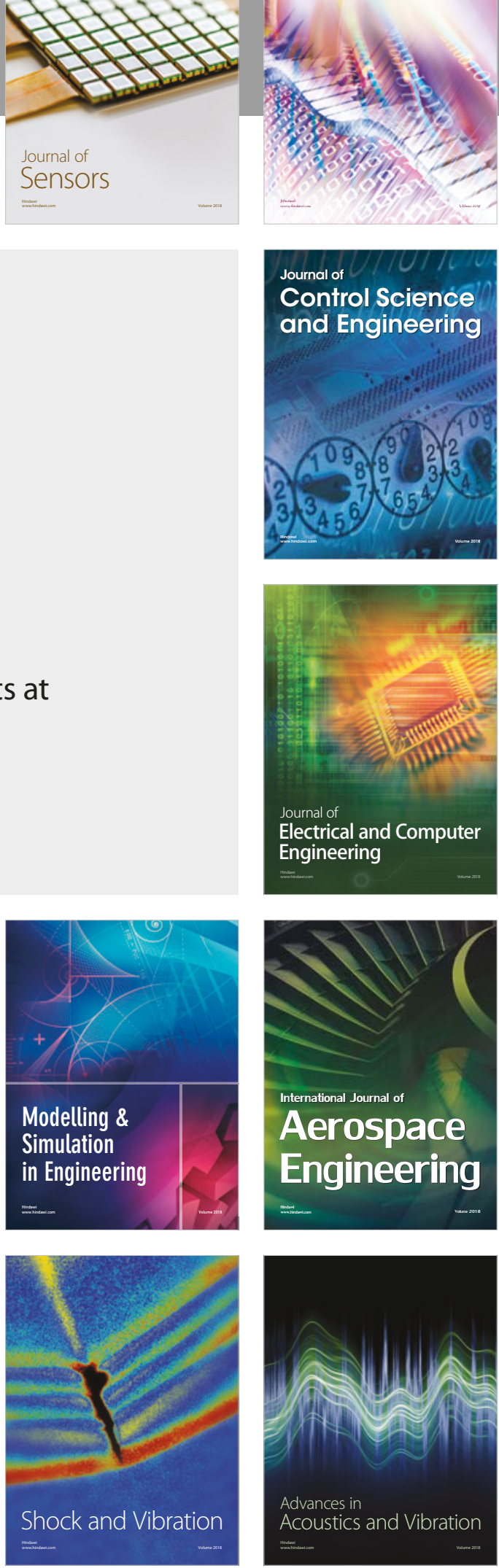